http://jmscr.igmpublication.org/home/

ISSN (e)-2347-176x ISSN (p) 2455-0450

crossref DOI: https://dx.doi.org/10.18535/jmscr/v7i7.173

Journal Of Medical Science And Clinical Research

IGM Publication

An Official Publication of IGM Publication

\title{
Clinicoetiological Study of Acute Flaccid Paralysis in Children under 15 Years Age Group
}

\author{
Authors \\ Dr Nita R. Sutay ${ }^{1}$, Dr Tejasi L. Sawant ${ }^{2}$ \\ ${ }^{1}$ Prof\& Head, Dept of Paediatrics, Grant Govt Medical College \& Sir JJ Group of hospitals, Mumbai \\ ${ }^{2}$ Resident Doctor, Grant Govt Medical College\& Sir JJ Group of hospitals, Mumbai
}

\begin{abstract}
Acute Flaccid Paralysis (AFP) is a complex clinical syndrome with broad array of potential etiologies and clinical features. The incidence of polio acute flaccidparalysis (AFP) has decreased in India but the non-polio AFP (NPAFP) rate has increased since 2000.It is useful to distinguish the possible causes of AFP in children using a neuro-anatomical approach. The accurate and early diagnosis of the cause is very important while managing the cases of AFP. This study is prospective and retrospective observational study. The objectives of our study were to study clinical features and etiology of AFP cases in children under 15 years age group.50 children under 15 years age group admitted in the tertiary care hospital from 2014 to 2018 with acute onset focal neurological weakness or paralysis were included in this study. Most common age group affected was 3 to 8 years (44\%). Guillain-Barré syndrome (GBS) was the most frequent final diagnosis (78\%) followed by Bell's palsy (8\%), Transverse Myelitis (6\%), Traumatic Neuritis (4\%) and others (4\%). NCV studies revealed Acute Inflammatory Demyelinating Polyneuropathy (AIDP) as most common variant (38\%) of GBS followed by Acute Motor Axonal Neuropathy (AMAN-31\%) and Acute Motor Sensory Axonal Neuropathy(AMSAN-10\%).51\% cases of GBS had history of antecedent infection while 54\% cases had cranial nerveinvolvement.CSF cytoalbumino dissociation was seen in only in $11 \%$ cases of GBS. This study concluded that GBS was the most common cause of AFP and AIDP was the most common variant of GBS.
\end{abstract}

\section{Introduction}

As per WHO, AFP defined as "any child under 15 years of age with acute onset of focal weakness or paralysis (including GBS)" or any person with paralytic illness at any age when polio suspected, characterised by flaccid (reduced tone), without other obvious causes (e.g. trauma). ${ }^{1}$

The incidence of polio acute flaccid paralysis (AFP) has decreased in India but the non-polio AFP (NPAFP) rate has increased since 2000.In India, NPAFP rate is 11.82 per 100000 population while the expected rate is 1 to 2 per
100000 population. $^{2}$ Anatomically AFP can be classified into 4 groups based on four levels of motor unit namely anterior horn cell, motor fibers, neuromuscular junction and muscles.

Poliovirus (polio) is a highly infectious, incurable disease that remains endemic in Afghanistan, Pakistan and Nigeria. ${ }^{3}$ The overall risk of vaccineassociated paralytic poliomyelitis in the United States and Latin America is one case per 2.5 million oral poliovirus vaccine doses. Nonpolio enteroviruses have been associated with polio-like paralytic disease. 
Guillain-Barre syndrome (GBS) is the most common cause of AFP in many parts of the world. ${ }^{4}$ GBS has worldwide incidence of 0.6-4 cases per 100,000 per year. ${ }^{5}$ Over 50 percent of AFP cases in both industrialized and developing countries are of GBS. ${ }^{6,7}$ GBS occurs in 50-70 percent of its patients within 2-28 days after a gastrointestinal or respiratory infection.

As a cause of AFP, acute transverse myelitisis less frequent than GBS or paralytic non polio enterovirus infection. Its annual incidence is less than one case per 2 million population. ${ }^{8}$ The common presentation includes initial phase of spinal shock, weakness of the lower extremities, AFP, urinary distention, constipation, hyporeflexia, sensory impairment, severe pain and paraesthesia. After 2-3 weeks hyperreflexia and spasticity appear.

Bell's palsy is the most common cause of facial paralysis during childhood. The prognosis for Bell's palsy is said to be generally better in children than in adults. Traumatic neuritis is suspected in cases in which there is one limb involvement with definite history of injection in that limb (usually less than $24 \mathrm{~h}$ ) before the onset of paralysis. Hypokalemic Paralysis is an important differential in any child particularly in a younger child with AFP.

It is useful to distinguish the possible causes of AFP in children using a neuro-anatomical approach. Information is derived from the history and neurological examination.

\section{Aims and Objectives}

Aims and objectives were to study the clinical features and etiology of acute flaccid paralysis in children under 15 years age group.

\section{Materials and Methods}

This study is prospective and retrospective observational study. 50 children under 15 years age group admitted in the tertiary care hospital from 2014 to 2018 with acute onset focal neurological weakness or paralysis were included in this study. Subjects fulfilling the inclusion criteria were enrolled in the study after approval from Institutional Ethics Committee. Subjects were enrolled after obtaining informed consent from lawful guardian. The presence of neurological deficit with onset more than 4 weeks, age more than 15 years were not included. This study also excludes patients with UMN (Upper Motor Neuron) diseases and non-progressive motor disorders (cerebral palsy). Patients with serum electrolyte abnormalities, deranged thyroid profile, deranged serum calcium were excluded if they found to be the underlying cause for neurological manifestations. Study subjects were investigated as per the clinical profile of the complaints and syndromic diagnostic premonition.

\section{Results}

The present study was a prospective and retrospective observational study to study the clinical features and differential diagnosis of AFP. The study group included 50 patients (35 males and 15 females) out of which $44 \%$ were in age group of 3-8 years (most common age group) and $92 \%$ of them born of non consanguineous marriage. All the subjects in the study presented with acute flaccid paralysis and most common clinical presentation was limb weakness (94\%) followed by muscle pain (44\%), facial weakness (24\%), sensory disturbances (18\%),breathing difficulty $(6 \%)$, swallowing difficulty $(4 \%)$.

In this study $51 \%$ cases who diagnosed as GBS had history of antecedent infection, out of which $60 \%$ had respiratory tract infections, $25 \%$ had gastrointestinal tract infection while in $15 \%$ cases source of infection was not known. Limb weakness at time of presentation in GBS cases was studied in detail on basis of clinical history and examination which concluded that $82 \%$ cases had involvement of all four limbs at time of presentation, $74 \%$ had symmetrical type of weakness and most common duration of weakness progression was 5-10 days. Study also revealed that $53 \%$ cases of GBS had involvement of cranial nerves, out of which $52 \%$ cases had facial palsy, $47 \%$ had bulbar palsy while multiple nerves involvement (VII, IX, X, XI) was seen in $38 \%$ cases. In present study of 39 cases of GBS, CSF 
analysis was done in 36 cases, out of which only $11 \%$ had CSF cytoalbuminodissociation.

In present study of 50 patients mortality was $28 \%$.Complications leading to death were studied in these cases which concluded that the most common complication leading to death was pneumonia (43\%) followed by sepsis $(21 \%)$, ARDS(14\%). Other causes include arrhythmias, venous thromboembolism, cardiac arrestetc. were seen in $14 \%$ cases whereas DIC in $7 \%$ cases.

Study revealed GBS as the most common cause of AFP, diagnosed in $78 \%$ cases followed by facial palsy $(8 \%)$, transverse myelitis $(6 \%)$, traumatic neuritis (4\%) and others (4\%).NCV studies in these GBS subjects concluded that AIDP was the most common variant of GBS(38\%),followed by AMAN(35\%),AMSAN(10\%), others(2\%).NCV studies of $13 \%$ cases did not specify any cause. Most common duration of hospital stay was 31-60 days (33\%) among AFP cases.

\section{Discussion}

In the present study of 50 patients, it was found that the most common cause of Acute Flaccid Paralysis (AFP) was Guillain Barre Syndrome (GBS). This finding correlates with the findings of retrospective study conducted by Gusharn Singh Narang et al (2011) where GBS found to be the leading cause in $55.9 \%$ of the patients. As per retrospective studies conducted by Rehman A, Idris $M$ et al (2007) Tsang RS, Valdivieso-Garcia et al (2003) and Tekgul, Sardaroglu Tekgul(2003), GBS found to be the most common cause of AFP. ${ }^{9,10,11}$ As per study conducted by Mohsin N, Asimi R. et al (2016), Molinero MR, Varon D et al (2003) and Mc Grogan A, Madle GC et al (2009) on AFP epidemiology, GBS found to be most common cause of AFP.

In present study AIDP was the most common subtype of GBS. This finding correlating with study conducted by Ali Zohair Nomani, Mansoor Iqbal et al (2015) which concluded that AIDP as the most common variant $(44 ; 57.8 \%)$ followed by AMAN $(19 ; 25 \%)$ and AMSAN $(7 ; 9.2 \%)$; axonal $($ AMAN + AMSAN $=26,34.2 \%) \cdot{ }^{12}$ Similar results were found from studies conducted by Zaheer et al. (2005), Siddiqui and colleagues (2013)and Yadegari et al (2014) ${ }^{13}$.

In present study $41.28 \%$ cases of GBS were associated with antecedent history of infection, most common was Respiratory Tract Infection (60\%) followed by Gastrointestinal Infection (25\%). This study correlates with studies conducted by Benjamin R Wakerley et al (2016), Valerie Sivadon Tardy et al (2006) and Udaya Seneviratne (2000) ${ }^{14}$.

Cranial nerve involvement in GBS is common and a well-known fact, however there are no studies only focused on cranial nerve in GBS. In present study cranial nerve involvement seen in $53 \%$ of GBS cases. Facial nerve was the most common nerve involved .Many of the studies done before quote variable involvement of cranial nerves ranging from $50 \%$ to $75 \%$, for example, Loeffel et $\mathrm{al}^{15}$ have quoted $50 \%$ and Dhadke et $\mathrm{al}^{16}$ had $62.5 \%$ involvement, which correlate well with our study.

In present study most common complication leading to mortality is pneumonia $(42.85 \%)$ followed by sepsis $(21.42 \%)$. In this study out of 50 patients of Acute Flaccid Paralysis, 14 patients died. This study correlating with the findings of the retrospective studies conducted by Archana B Netto et al (2011), Ng KK, Howard RS (1995) and Alshekhlee A, Hussain Z et al $(2008)^{17}$

As per study conducted by Taly et al mortality rate was $38.3 \%$. Out of 153 patients of Guillain Barre Syndrome (GBS) seen over 5.5 years, there were 47 (M: F 38.9) critically ill patients. Amongst complications pulmonary complications were the most common. ${ }^{18}$

In present study CSF cytoalbuminodissociation was seen in $12.5 \%$.According to Yadegari and colleagues, higher levels of CSF protein are more frequent in AIDP subtype. This study also correlates with study conducted by Nomani, Ali Zohair; Iqbal et al (2015) where cytoalbuminological dissociation found to be more frequent in $\operatorname{AIDP}(61.7 \%){ }^{19}$

In the present study most common symptom with which GBS cases presented to health care centre was symmetrical limb weakness seen in $74 \%$ 
cases. This finding correlates with studies conducted by Asbury AK, Cornblath et al (1990), Fokee C, Van den Berg B et al (2014), Sejvar JJ, Kohl KS et al (2011), Van Doorn PA (2013). ${ }^{20}$

In present study, majority of AFP cases belonged to age group of 3 to 8 years (44\%). This finding correlates with study done by Preet Khona et al (2016). In present study males outnumbered females where $70 \%$ were males. Male predominance was also seen in study conducted by Shah FU, Salih et al (2002) and Naveed Mohsin et al.

\section{Conclusion}

Present study revealed that the accurate and early diagnosis of the cause is very important while managing the cases of AFP. Detailed history and clinical examination is the mainstay for the clinical diagnosis of AFP. This study concluded that GBS was the most common cause of AFP and AIDP was the most common variant of GBS. Respiratory tract infections were the most common antecedent infection in GBS cases. Facial nerve was the most common nerve involved in GBS cases while pneumonia was the most common complication leading to death.

\section{References}

1. Saraswathy TS, Zahrin HN, Apandi MY, Kurup D, Rohani J, Zainah S, Khairullah NS. Acute flaccid paralysis surveillance: looking beyond the global poliomyelitiseradication initiative. Southeast Asian J Trop Med Public Health. 2008 Nov1;39(6):1033-9

2. Vashisht N, Puliyel J, Sreenivas V. Trends in nonpolio acute flaccid paralysis incidence in India 2000 to 2013. Pediatrics. 2015 Feb 1;135(Supplement1): S16-7

3. Ahmad A, Rehman A. one year surveillance data of acute flaccid paralysis at Bahawal Victoria Hospital Bahawalpur. PAKISTAN JOURNAL OF MEDICAL SCIENCES. 2007 Apr 1;23(3):308
4. Jones Jr HR. Topical Review: Childhood Guillain-Barré Syndrome: Clinical Presentation, Diagnosis, and Therapy. Journal of Child Neurology. 1996 Jan;11(1):4-12.

5. Hughes RA, Rees JH. Clinical and epidemiologic features of Guillain-Barré syndrome. Journal of Infectious Diseases. 1997 Dec 1;176(Supplement_2):S928.

6. Andrus JK, De Quadros C, Olivé JM, Hull HF. Screening of cases of acute flaccid paralysis for poliomyelitis eradication: ways to improve specificity. Bulletin of the World Health Organization. 1992;70(5):591.

7. Olivé JM, Castillo C, Castro RG, de Quadros CA. Epidemiologic study of Guillain-Barré syndrome in children $<15$ years of age in Latin America. The Journal of infectious diseases. 1997 Feb 1;175(Supplement_1):S160-4

8. Hizarcıoğlu M, Gülez P, Ünalp A, Gülhan T, Uran N. Early-onset acute partialtransverse myelitis: case report. İzmir Dr.Behçet Uz ÇocukHastanesi Dergisi.2013;3(2):133-7.

9. Anis-ur-Rehman IM, Elahi M, Jamshed AA. Guillain Barre syndrome: Theleading cause of acute flaccid paralysis in Hazara division. J Ayub Med CollAbbottabad. 2007;19(1):1.

10. Atassi MZ, Casali P, Atassi MZ, Casali P. Molecular mechanisms of autoimmunity. Autoimmunity. 2008 Jan 1; 41(2):123-32.

11. Tekgul H, Serdaroglu G, Tutuncuoglu S. Outcome of axonal and demyelinating forms of Guillain-Barré syndrome in children. Pediatric neurology. 2003 Apr1;28(4):295-9.

12. Nomani AZ, Iqbal M, Majeed H, Badshah M, Nabi S, Jan Z, Jamil U. Albuminocytological dissociation in different electrophysiological gbs variants. Pakistan Journal of Neurological Sciences (PJNS). 2015;10(4):32-6. 
13. Yadegari S, Nafissi S, Kazemi N. Comparison of electrophysiological findingsin axonal and demyelinating Guillain-Barre syndrome. Iranian journal ofneurology. 2014 Jul 4;13(3):138.

14. Sharma KS, Singh R, Shah GS. Guillain Barre Syndrome: Major Cause of Acute Flaccid Paralysis in Children and Adolescents of Nepal. Journal of Nepal Paediatric Society. 2011 May 1;31(2).

15. Löffel NB, Rossi LN, Mumenthaler M, Lütschg J, Ludin HP. The Landry Guillain-Barré syndrome: Complications, prognosis and natural history in 123 cases. Journal of the neurological Sciences. 1977 Aug 1;33(1):71-9.

16. Dhadke SV, Dhadke VN, Bangar SS, Korade MB. Clinical profile of Guillain Barre syndrome. J Asso Physicians India. 2013 Mar;61:168-72.

17. Alshekhlee A, Hussain Z, Sultan B, Katirji B. Guillain-Barré syndrome: incidence and mortality rates in US hospitals. Neurology. 2008 Apr29;70(18):1608-13.

18. Taly AB, Gupta SK, Vasanth A, Suresh TG, Rao U, Nagaraja D, Swamy HS, Rao S, Subbakrishna DK. Critically ill Guillain Barre syndrome. The Journal ofthe Association of Physicians of India. 1994 Nov;42(11):871-4.

19. Nomani AZ, Iqbal M, Majeed H, Badshah M, Nabi S, Jan Z, Jamil U. Albuminocytological dissociation in different electrophysiological gbs variants. Pakistan Journal of Neurological Sciences (PJNS). 2015;10(4):32-6.
20. Asbury AK, Cornblath DR. Assessment of current diagnostic criteria for Guillain- Barré syndrome. Annals of Neurology: Official Journal of the American Neurological Association and the Child Neurology Society. 1990;27(S1): S21-4. 